\title{
Passive and Active Reconfigurable Scan-Beam Hollow Patch Reflectarray Antennas
}

\author{
M. Hajian, ${ }^{1}$ B. Kuijpers, ${ }^{2}$ K. Buisman, ${ }^{2}$ A. Akhnoukh, ${ }^{2}$ M. Pelk, ${ }^{2}$ \\ L. C. N. de Vreede, ${ }^{2}$ J. Zijdeveld, ${ }^{3}$ L. P. Ligthart, ${ }^{3}$ and C. Spitas ${ }^{1}$ \\ ${ }^{1}$ Faculty of Industrial Design, Delft University of Technology, Landbergstraat 15, 2628 CE Delft, The Netherlands \\ ${ }^{2}$ Department for Electrical Engineering, DIMES, Delft University of Technology, Mekelweg 4, 2628 CD Delft, The Netherlands \\ ${ }^{3}$ Microwave and Radar Technologies, Department for Electrical Engineering, Delft University of Technology, Mekelweg 4, \\ 2628 CD Delft, The Netherlands
}

Correspondence should be addressed to B. Kuijpers, bjkuijpers@gmail.com

Received 28 December 2011; Accepted 30 January 2012

Academic Editors: C. Beckman and W. C. van Etten

Copyright ( $) 2012$ M. Hajian et al. This is an open access article distributed under the Creative Commons Attribution License, which permits unrestricted use, distribution, and reproduction in any medium, provided the original work is properly cited.

\begin{abstract}
The design concept of passive and active reconfigurable reflectarray antennas has been proposed and tested. The antenna elements in the array are identical hollowed patches. In the first phase of study the slots are loaded with a SMD capacitor to set the required phase shift needed for array implementation. Simulations show promising results. Mounting a SMD capacitor in such a configuration can be considered as the first step in using capacitive loading on a slotted patch for active microstrip reflectarrays. It is shown that by adjusting the capacitance values it is possible to scan the beam. In the second phase, the patch elements are loaded with active varactor-diode device which its reflected phase can be varied. This phase alteration is based on the variation of the diode capacitance which can be achieved by varying the biasing voltage of the active varactor device. In latter approach by activating these varactor devices, the phase of each antenna element in the array configuration can be adopted dynamically and consequently, its beam direction can be reconfigured. The reflectarrays incorporating passive and active elements have been built and tested at $7.0 \mathrm{GHz}$ and $6.0 \mathrm{GHz}$, respectively. The performance of the proposed reconfigurable antennas is excellent, and there is good agreement between the theoretical and measurement results which pioneers design of arbitrarily reconfigurable antennas.
\end{abstract}

\section{Introduction}

In the past decade the research and development on analytical and experimental techniques for Microstrip ReflectArray (MRA) antennas have received a considerable attention. This is due to the their low-cost production, low-weight, and very thin flat leading to a excellent alternative to the traditional reflector antennas. The main objective of recent studies is the development of active reflectarrays. In this paper the passive and active phase shifting techniques for hollowed microstrip antenna elements loaded with Surface Mounted Device (SMD) capacitor and varactor-diode device, respectively, are addressed. There are different types of phasing techniques reported in the literature: stubs $[1,2]$, where patch antenna elements are loaded with different stubs length, rotation [3], where patch antenna elements are rotated, and variable size $[4,5]$, where the resonance length of the antenna is adjusted. These conventional methods require alternation of the patch elements geometry in an array. The phase shift technique proposed in this paper is capacitive loading. The antenna element is hollowed patch antenna [6]. The passive and active phasing techniques addressed in this paper provide a unique approach using a capacitive load across the slot of the patch to alter its phase. Different values of the capacitance lead to a change in the reflected phase. In the first stage, the passive phasing technique is achieved by loading the antenna element with SMD capacitor. This is the preliminary study to design and measure the reflected phased array antenna using active capacitive loading. In the second stage, the results of the first phase are used to design the phasing technique by loading the antenna elements with varactor diode. In this case the capacitance is altered using 
different biasing. The varactor chips have been designed and developed at Delft Institute for Micro-Electronics and Submicron technology (DIMES) and act as tunable capacitive devices. The fundamental theory and design of the varactor are addressed in $[7,8]$. The electronically tunable MRA provides advantages over the active phased array due to elimination of a beamforming network, which can be complex and lossy for a high-directivity antenna and the need of less active components. The initial approach was to imitate the electrical behaviour of the hollow patch with variable slot width by integrating active switches over the slot. The width of the slot is then adaptively reconfigured using the switches. The active switch is a set of microelectromechanical systems (MEMS) switches, which can monolithically be integrated and packaged onto the same substrate. Previous works also have shown that it is possible to reconfigure the beam of a reflectarray antenna mechanically but also electrically using MEMS [9-11]. However, employing such an approach leads to a more complex system since for continuously reconfiguring the beam, the required number of MEMS would increase substantially. Moreover, the beam steering would be discrete and not continuous. The authors in [10] have proposed a very complex design MEMS-based structure patch resonators with adjustable height. The patterns show a rather poor performance. In [12], the authors suggest a technology using patch aperture coupled to a transmission line loaded with two diodes. The total impedance of the transmission line can be varied by changing the reverse bias voltage applied to diodes and hence creating a phase variation in the reflection coefficient. There the effect of higher intermodulation caused by the diode and surface waves due to the thick substrata is not addressed. Moreover, the sensitivity of the design is very high.

In this paper a more simpler approach is taken. Prototype passive and active MRA antennas loaded with SMD capacitor and active varactor-diode are designed, build, and measured to demonstrate the suggested phasing technique. The rest of this paper is organized as follows. In Section 2, the geometries of antenna elements are given. The phase diagrams of the passive and active antenna elements are studied in Section 3. In Section 4 the design and layout of varactor chip are presented. To demonstrate the beam scanning concept using the suggested phasing technique, Section 5 addresses the design and simulation results of MRA antennas. In Section 6, the measurement results of array antennas are presented. Finally, in Section 7 conclusions are given.

\section{Geometry of SMD and Varactor-Diode-Hollowed Patch}

In this section the geometry and design parameters of passive and active antenna elements are given. For the design of the array antennas the phase diagram of a single element is used. To demonstrate the correctness of this approach, the author in [13] has compared the phase diagrams of a single element with an array of $2 \times 2$ elements of variablesized as function of resonance length at different frequencies. The other parameters for both configurations were kept the

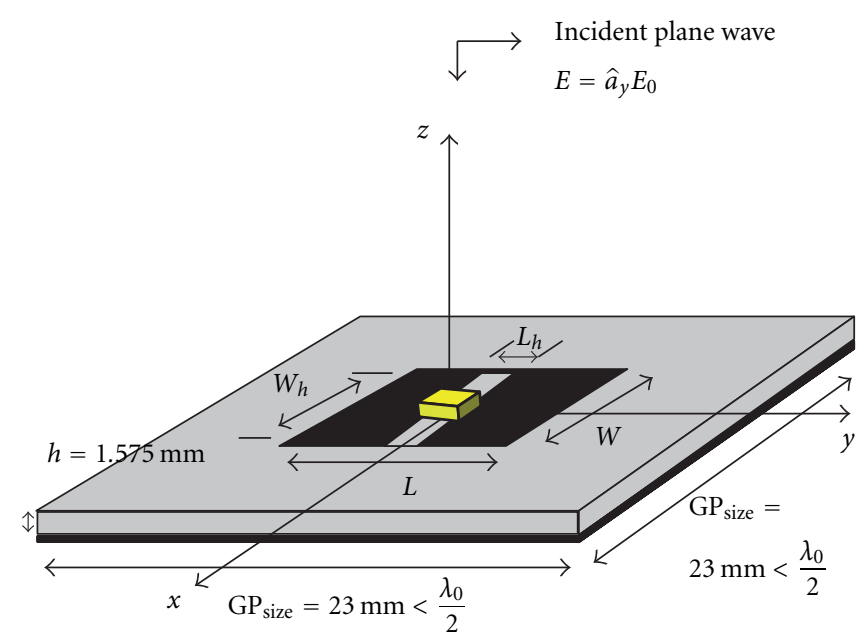

SMD capacitor or active varactor-diode

Substrate

Metal

Figure 1: Geometry of a single element.

same. It was shown that there was an excellent agreement between the two approaches and there was no difference between phase of a single element and the array. Moreover, the phase of the single element is compared with the results shown in [4]. The authors in [4] have used the finite array approach using an $N$-elements array to determine the phase diagram. The results between both approaches were similar. Simulation results have validated that a single element analysis is sufficient for array design.

2.1. Passive. The geometry of the single element for passive hollowed patch antennas loaded with capacitor is shown in Figure 1.

The geometry consists of a metal-slotted patch on a grounded substrate. The SMD is placed at the center of the slot. The antenna is illuminated by a linear horizontally polarized plane wave in the positive $y$-direction. The single element is designed using a commercial package based on the full-wave solution Method of Moments (MoM). Table 1 shows an overview of the parameters used for the design. The SMD type is a standard ceramic capacitor, 0805 series [14].

2.2. Active. The geometry of elementary radiator consisting of a hollow patch loaded with the varactor is depicted in Figure 2.

For integrating the varactor chip, there is an island introduced in the mid del of the path [13]. The system parameters are presented in Table 2 . The central part of the patch consists of the varactor chip with an additional metalized plate, which two vias are used to transfer the Bias and GND control signals between the two sides of the PCB. The connection between points $A$ and $B$ through the chip represents the RF path. The chip can be mounted on top of the metal plate in the center of the slot. The chip has five connection points on top which 
TABle 1: Parameters of the passive hollow patch loaded with SMD capacitor.

\begin{tabular}{lc}
\hline Parameter & Value \\
\hline Operating frequency & $f=7.0 \mathrm{GHz}$ \\
Substrate thickness & $h=1.575 \mathrm{~mm}$ \\
Substrate permittivity & $\epsilon_{r}=2.33$ \\
Loss tangent & $\tan \delta=0.0005$ \\
Patch width, length & $W=15 \mathrm{~mm}, L=12 \mathrm{~mm}$ \\
Slot width, length & $W_{h}=13 \mathrm{~mm}, L_{h}=0.55 \mathrm{~mm}$ \\
Substrate width, length & $\lambda_{0} / 2, \lambda_{0} / 2 \mathrm{~mm}$ \\
SMD 0805 thickness & $H_{\text {smd }}=0.8 \mathrm{~mm}$ \\
SMD 0805 width & $L_{\text {smd }}=2.0 \mathrm{~mm}$ \\
SMD 0805 metal connection length & $L_{2 \mathrm{smd}}=0.35 \mathrm{~mm}$ \\
Resonance capacitance & $C_{0}=0.55 \sim 0.6 \mathrm{pF}$ \\
\hline
\end{tabular}

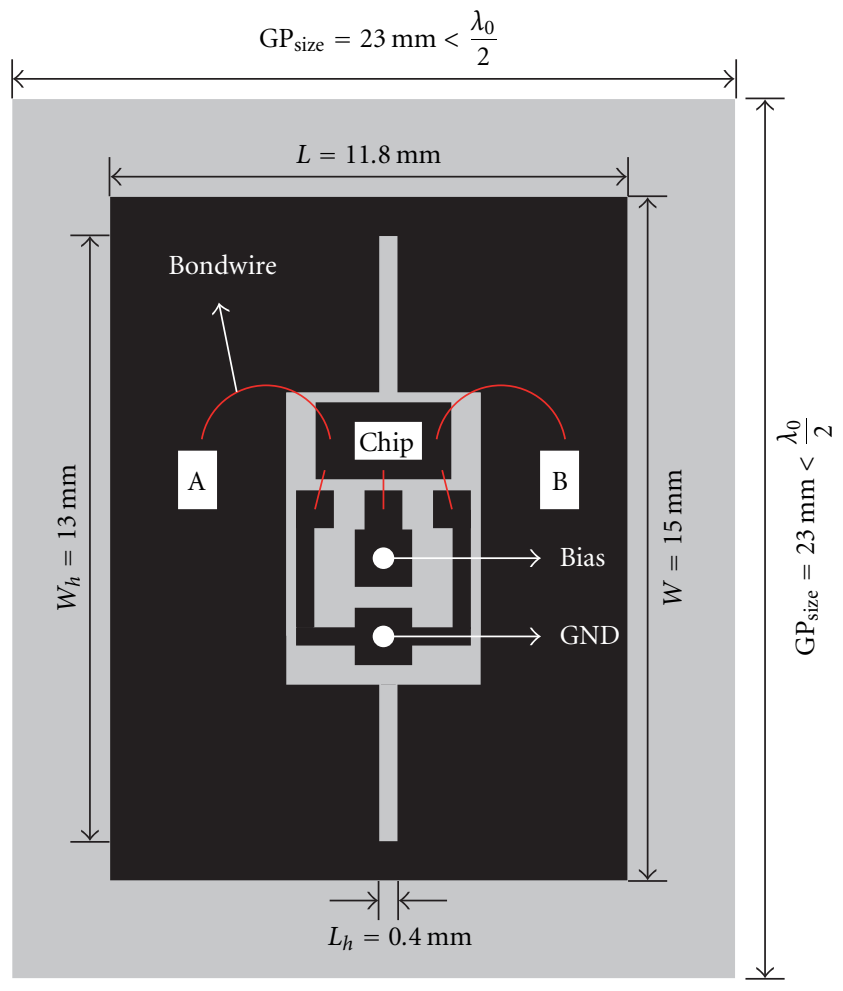

FIGURE 2: Geometry of the varactor-loaded hollow patch.

are wired to the patch laminate using bondwires. On the bottom, the patch consists of a groundplane with islands directly under the center of the chip. To ensure decoupling of the control signal from the RF signal, a SMD capacitor is integrated on the bottom of the patch.

The choice of different operational frequencies for active and passive is due to availability of SMD capacitor and varactor.
TABLE 2: Parameters of the varactor-loaded hollow patch.

\begin{tabular}{lc}
\hline Parameter & Value \\
\hline Operating frequency & $f=6.0 \mathrm{GHz}$ \\
Substrate thickness & $h=1.575 \mathrm{~mm}$ \\
Substrate permittivity & $\epsilon_{r}=2.45$ \\
Loss tangent & $\tan \delta=0.0012$ \\
Patch width, length & $W=15 \mathrm{~mm}, L=11.8 \mathrm{~mm}$ \\
Slot width, length & $W_{h}=13 \mathrm{~mm}, L_{h}=0.4 \mathrm{~mm}$ \\
Substrate width, length & $\lambda_{0} / 2, \lambda_{0} / 2 \mathrm{~mm}$ \\
Chip thickness & $0.5 \mathrm{~mm}$ \\
Chip width & $0.65 \mathrm{~mm}$ \\
Chip length & $0.9 \mathrm{~mm}$ \\
Resonance capacitance & $C_{0}=0.55 \sim 0.6 \mathrm{pF}$ \\
\hline
\end{tabular}

\section{Phase Diagram}

In this section the phase diagrams of passive and active capacitive loaded hollowed patch antennas are presented. In [13] the effects of the design parameters on the phase diagram have been studied and will not be addressed here.

3.1. Passive. Figure 3(a) shows the reflected phase of a single element as a function of the SMD capacitance. The phase curves show the very limited bandwidth. A shift between 6.87.2 GHz already would greatly influence the array behaviour. The affect on the phase behaviour is most dominant for the low SMD values. Maximum sensitivity between $1.0-3.3 \mathrm{pF}$ is about $160^{\circ} / \mathrm{pF}$. This is an indication that the sensitivity of the system is a crucial part in the usage of SMD for design of reflectarray. With a tolerance for example of $\pm 0.25 \mathrm{pF}$ the phase for an element can be deviated by $40^{\circ}$. Figure 3(b) shows the reflected phase as function of the frequency for different values of the capacitance. At $7.0 \mathrm{GHz}$ the SMD values between $1.0-3.9 \mathrm{pF}$ have a phase range of $200^{\circ}$. A wider range in SMD values especially in the lower region would increase this phase range. Decreasing the substrate thickness also increases the phase range but at the cost of sensitivity.

3.2. Active. Figure 4(a) shows the reflected phase of hollowed patch loaded with varactor-based diode as function of the capacitance at different frequencies. The phase range for a varying capacitance $0.3 \mathrm{pF} \leq C \leq 0.8 \mathrm{pF}$ is about $250^{\circ}$ with a maximum sensitivity of $40^{\circ}$ per $0.05 \mathrm{pF}\left(40^{\circ} / 0.05 \mathrm{pF}\right)$. A bias voltage from -12 to $0 \mathrm{~V}$ leads to a controllability of $0.05 \mathrm{pF} /$ Volt for this range, resulting in a maximum sensitivity to the control signal of about $40^{\circ} /$ Volt. The performance of the phase curves at frequencies $f=5.8 \mathrm{GHz}$ and $f=6.2 \mathrm{GHz}$ is deteriorated which demonstrates a narrow band behaviour of the antenna. Figure 4(b) depicts the frequency response of the phase diagram for different values of the capacitance. The resonance frequency is shifted to higher frequencies as $C$ decreases. This leads to the 


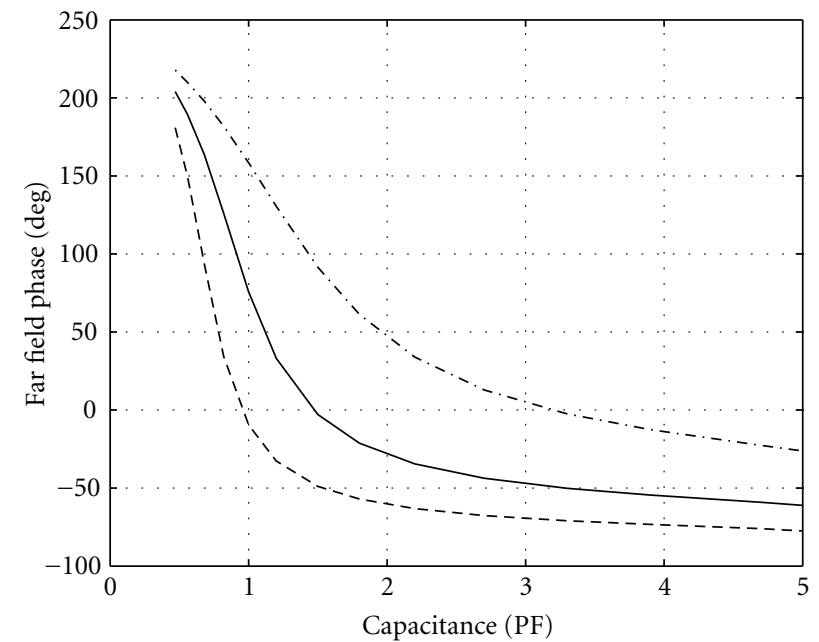

$-\cdot 6.8 \mathrm{GHz}$

$-7 \mathrm{GHz}$

$---7.2 \mathrm{GHz}$

(a)

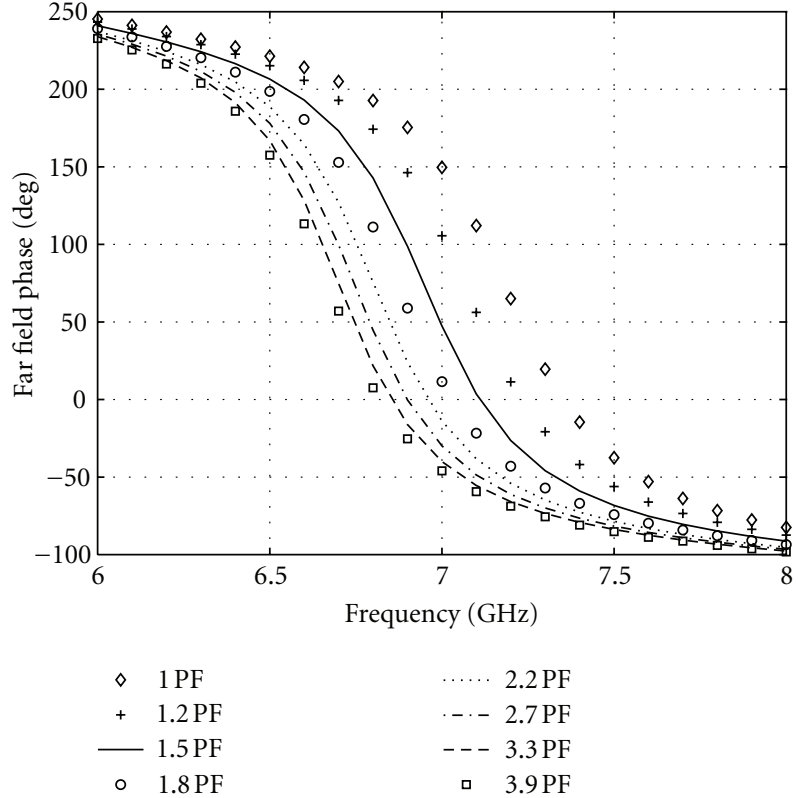

(b)

FIgURE 3: Phase diagram of a SMD capacitor-loaded hollowed patch as function of (a) capacitor; (b) frequency.

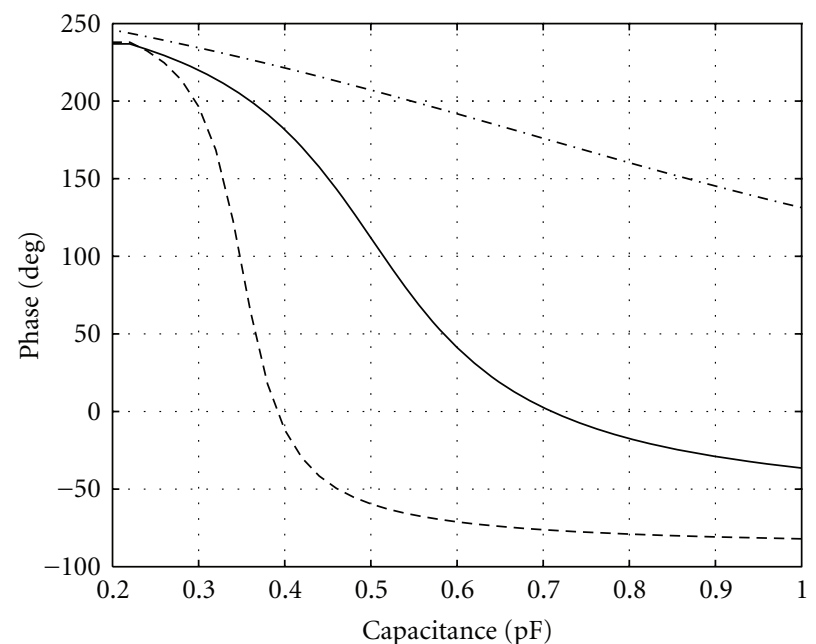

$\begin{aligned}-f & =5.8 \mathrm{GHz} \\ -f & =6 \mathrm{GHz}\end{aligned}$

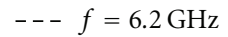

$L=11.8 \mathrm{~mm}$

(a)

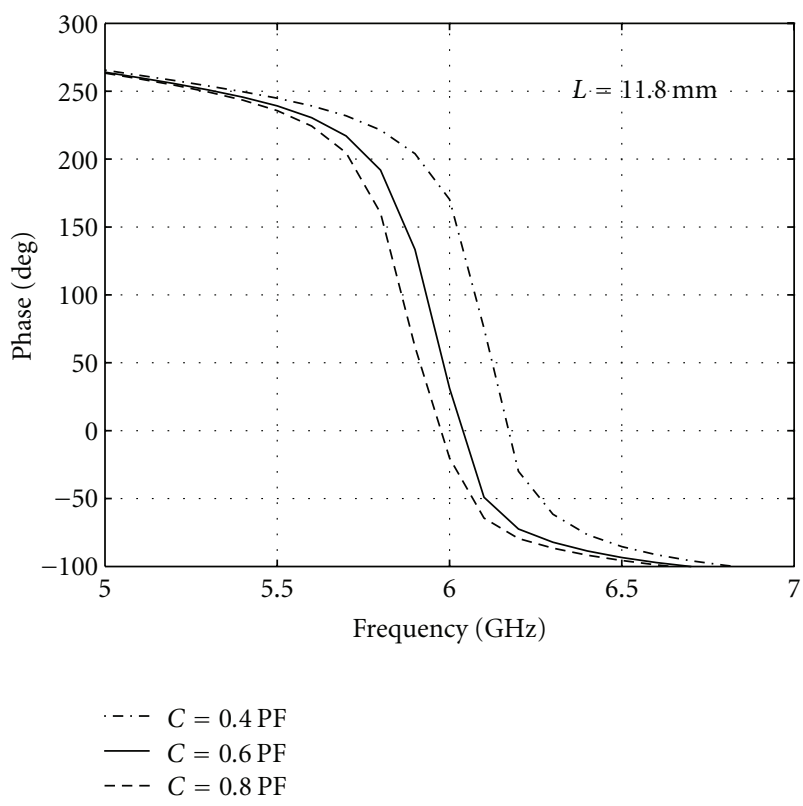

(b)

FIgURE 4: Phase diagram of a hollow patch loaded with tunable varactor as function of (a) capacitance, (b) frequency.

fundamental property that relates the change in capacitance to the shift in resonance frequency according to:

$$
C \uparrow \Longrightarrow \downarrow f_{\text {res }} .
$$

An increase in the capacitor value $C$ causes a decrease in the resonance frequency $f_{\text {res }}$. The phase curves indicate a small frequency band in which the resonance is shifted. The maximum shift in frequency for a $0.4 \mathrm{pF} \leq C \leq 0.8 \mathrm{pF}$ varactor capacitor range is $200 \mathrm{MHz}$.

\section{Varactors}

The tunable varactors are designed and developed at DIMES. It uses diode-based circuit topologies, which can act as high$Q$ “distortion-free" tunable capacitive elements. These diodes 


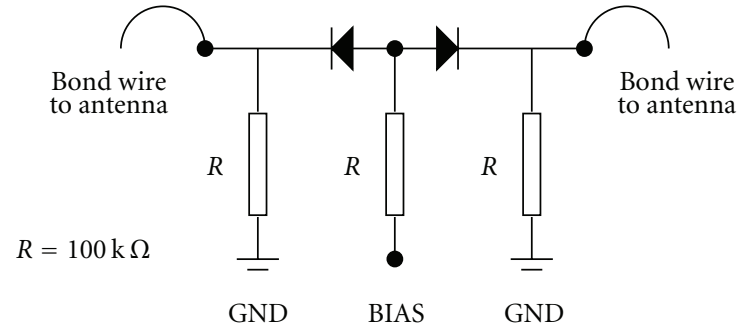

FIGURE 5: Varactor diodes devices topology.

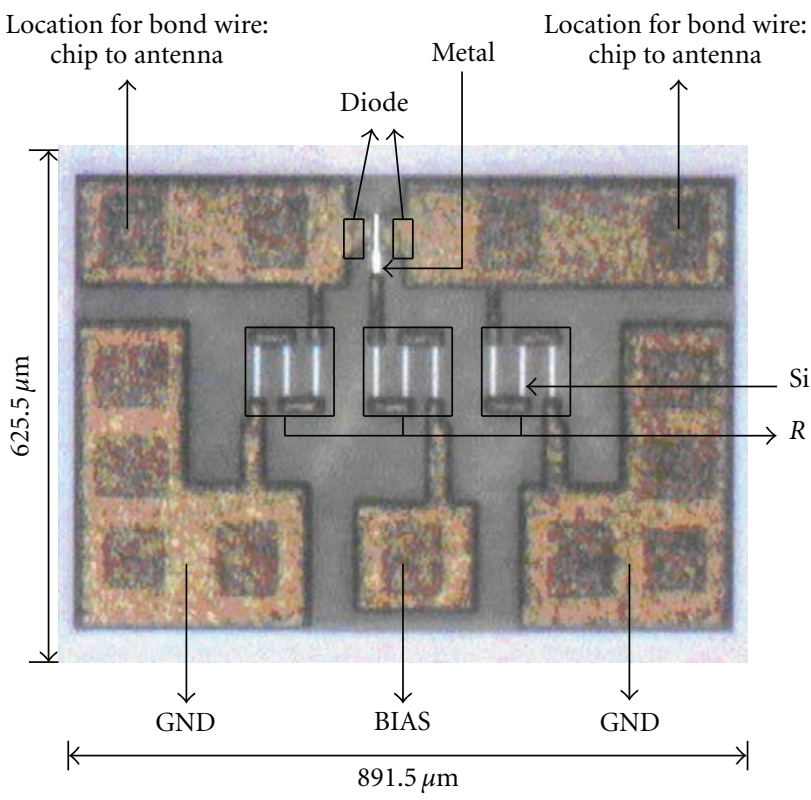

FIGURE 6: The layout of the varactor device.

are implemented in a novel ultra low-loss Silicon on Glass (SoG) technology and result in measured $Q_{s}$ of over 200 at $2 \mathrm{GHz}$. The diode configuration in Figure 5 can be applied to realize a voltage-controlled variable capacitor $[7,8]$. Due to the nonlinear behaviour of single diode which leads to higher order distortion responses, a diode configuration as in Figure 5 has been employed. Theoretically, this configuration is free from distortion. It has been demonstrated that for this topology the measured third-order distortion, IM3, shows an improvement compared to the traditional single varactor tuning more than $30 \mathrm{~dB}$ [7]. Another example of a moretraditional approach is the MEMS capacitor, which in its most popular implementation, is able to switch between two fixed capacitance values $[15,16]$. MEMS capacitors provide a high $Q$ at moderate-capacitance values, but they require nonstandard processing, and expensive packaging techniques, high-control voltages, and their reliability and switching speed are still poor compared to semiconductorbased solutions [7]. Other tuning techniques are based on voltage-variable dielectrics but exhibit similar drawbacks of manufacturability and performance [17]. Figure 6 represents the detailed layout of the varactor chip processed at DIMES. These Low Distortion Varactor Stack (LDVS) components
TABLE 3: SMD capacitances for $\theta_{\text {scan }}=5^{\circ}$ and $\theta_{\text {scan }}=15^{\circ}$ of $1 \times 5$ linear array.

\begin{tabular}{lcc}
\hline Array & Capacitance values per element & Scan angle \\
\hline $\mathrm{A}$ & $(1.5-1.8-2.2-2.7-3.9) \mathrm{pF}$ & $+5^{\circ}$ \\
$\mathrm{B}$ & $(1.0-1.2-1.5-2.2-3.9) \mathrm{pF}$ & $+15^{\circ}$ \\
\hline
\end{tabular}

TABLE 4: Parameters for active MRA antenna.

\begin{tabular}{lc}
\hline Frequency & $6.0 \mathrm{GHz}$ \\
Element spacing & $d_{y}=23.0 \mathrm{~mm}$ \\
Linear array & $1 \times 6$ Elements \\
Patch dimensions & $15.0 \mathrm{~mm} \times 11.8 \mathrm{~mm}$ \\
Slot & $13.0 \mathrm{~mm} \times 0.4 \mathrm{~mm}$ \\
Varactor range & $0.37 \mathrm{pF} \leq C \leq 0.98 \mathrm{pF}$ \\
Substrate thickness & $h=1.575 \mathrm{~mm}$ \\
Dielectric permittivity & $\epsilon_{r}=2.45$ \\
$\tan \delta$ & $5 e-04$ \\
\hline
\end{tabular}

are, due to their easy implementation and inherently high performance, suitable for use in a variety of high- $Q$ tunable circuits, including filters, switches, phase shifters, and matching networks [18]. Figure 7 depicts the frequency response and $C-V$ measurement results of varactor device.

\section{Array Simulation Results}

In this section the design and simulation results of beam scanning of the capacitive loaded MRA antenna are addressed. Amplitude tapering is not considered and it is not attempted to optimizer the radiation pattern, nor to study pattern synthesis. The main objective is to demonstrate the concept of beam scanning using the suggested phasing technique is demonstrated.

5.1. SMD-Based Scanning Capabilities. Due to the limited obtainable values of commercial SMD only a few arrays are designed. Normally the designer chooses a progressive phase and configures each element appropriately. The phase value of each element using SMD capacitors can only be set at discrete values. A scan angle can be selected between the $-30^{\circ}$ and $+30^{\circ}$ due to the limited phase range. In this paper the results are limited to arrays using commercially available, easy to mount SMD capacitors. Hence, the arrays can be built without demanding specialist assembly. Table 3 gives the capacitance values of the SMD loaded on the elements of $1 \times$ 5 linear array antenna. Figure 8 shows the simulation results for the different array configurations. The results illustrate the possibility of beam switching using capacitive loading.

5.2. Varactor-Based Scanning Capabilities. In order to demonstrate the concept of beam scanning an $1 \times 6$ uniform linear array is implemented and simulated in a MoM-based Electromagnetic simulator. Tables 4 and 5 present the array parameters. In Table 5, A to F indicates the capacitance values of the varactor chip. Due to the complexity of the design, it was decided to scan the beam only in the E-plane. For a controllable varactor with $0.37 \mathrm{pF} \leq C \leq 0.98 \mathrm{pF}$ the beam 


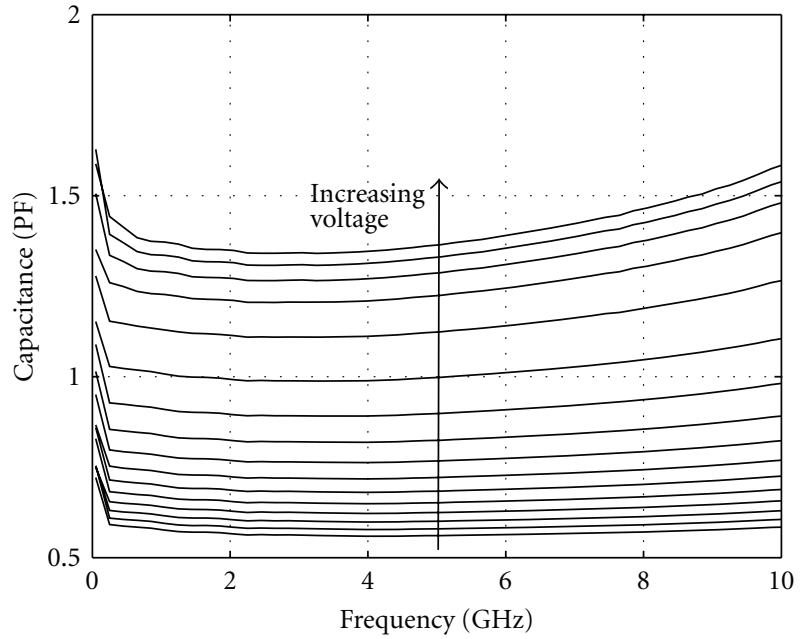

(a)

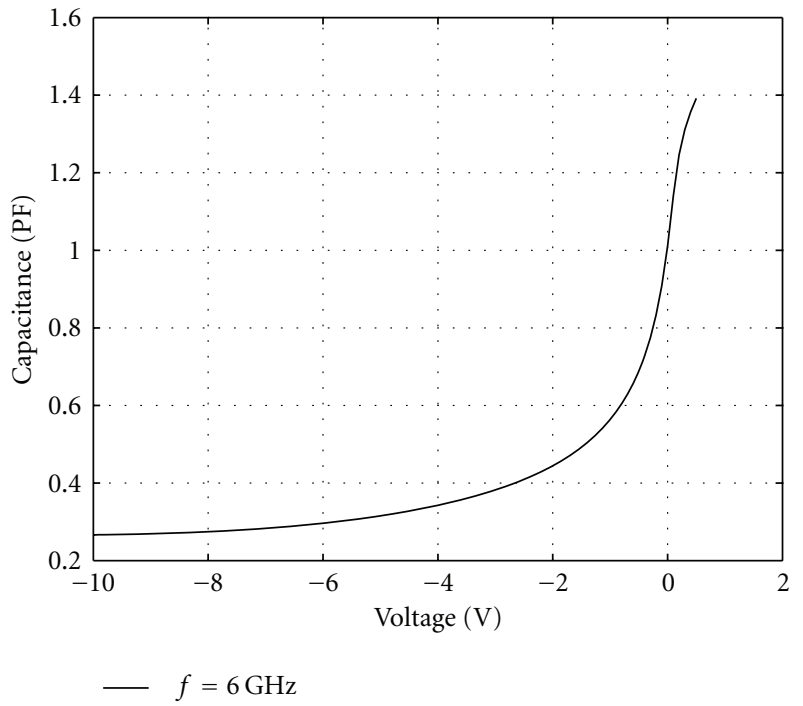

(b)

FIGURE 7: Measured characteristics of varactor device integrated into the antennas as function of (a) frequency; (b) voltage at 6.0 GHz.

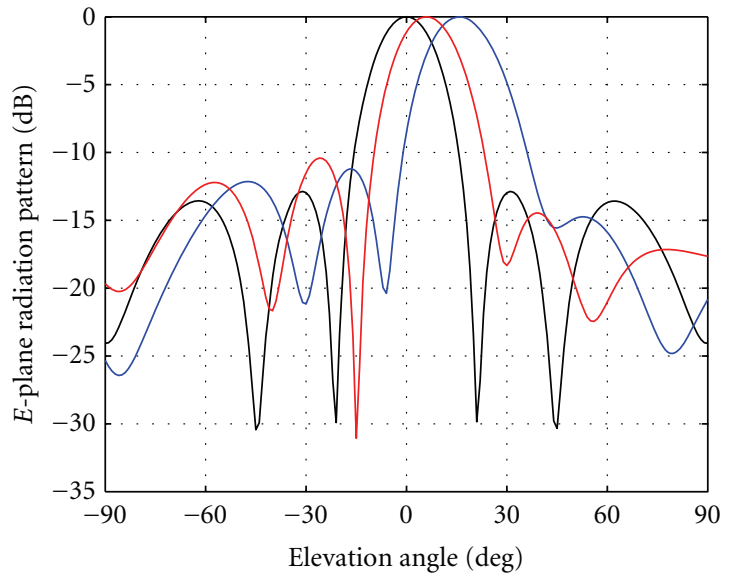

FIGURE 8: Simulated results for an $1 \times 5$ linear array antenna loaded with SMD capacitor.

can be scanned from $-15^{\circ} \leq \theta_{\text {scan }} \leq 15^{\circ}$. The controllability, however, must be with accuracies in the order of $0.02 \mathrm{pF}$. Figure 9 presents the simulation results for the different array configurations and hence demonstrating the potential of our concept. The scanning range can be increased using $1 \times 6$ linear array, that is, for $0.2 \mathrm{pF} \leq C \leq 1.0 \mathrm{pF}$ the beam can be scanned from $-22^{\circ}$ to $22^{\circ}$. The controllability, however, must be with accuracies in the order of $0.01 \mathrm{pF}$.

\section{Measurement Results}

In this section the manufactured antennas and the measurement results for the passive and active array antennas are presented.

6.1. Passive. A $4 \times 4$ and $5 \times 5$ antenna arrays are manufactured and their radiation pattern are measured. Distance between the antenna elements is $\lambda_{0} / 2$. The substrate material
TABle 5: Varactors capacitance for an $1 \times 6$ linear array, scanning $-15^{\circ} \leq \theta \leq 15^{\circ}$.

\begin{tabular}{lcc}
\hline Array & Capacitance values per element & Scan angle \\
\hline A: & $(0.37-0.45-0.50-0.560-0.660-0.980) \mathrm{pF}$ & $+15^{\circ}$ \\
B: & $(0.40-0.44-0.48-0.515-0.557-0.612) \mathrm{pF}$ & $+10^{\circ}$ \\
C: & $(0.47-0.50-0.52-0.570-0.650-0.730) \mathrm{pF}$ & $+5^{\circ}$ \\
D: & $(0.730-0.650-0.570-0.52-0.50-0.47) \mathrm{pF}$ & $-5^{\circ}$ \\
E: & $(0.612-0.557-0.515-0.48-0.44-0.40) \mathrm{pF}$ & $-10^{\circ}$ \\
F: & $(0.980-0.660-0.560-0.50-0.45-0.37) \mathrm{pF}$ & $-15^{\circ}$ \\
\hline
\end{tabular}

TABLE 6: SMD capacitor values mounted on different arrays.

\begin{tabular}{cccccc}
\hline Array & Col. 1 & Col. 2 & Col. 3 & Col. 4 & Col. 5 \\
\hline $5 \times 5$ & $0.47 \mathrm{pF}$ & $0.56 \mathrm{pF}$ & $0.68 \mathrm{pF}$ & $0.82 \mathrm{pF}$ & $1.0 \mathrm{pF}$ \\
$4 \times 4$ & $0.47 \mathrm{pF}$ & $0.56 \mathrm{pF}$ & $0.68 \mathrm{pF}$ & $0.82 \mathrm{pF}$ & \\
\hline
\end{tabular}

is RT/Duroid high frequency laminate with relative permittivity of $\epsilon_{r}=2.33$. Each element is manually integrated with a standard SMD capacitor. Figure 10 shows the manufactured arrays. The SMDs are visible in the centre of each patch. SMD values are specified in Table 6. For tuning the ground plane dimensions are altered during the measurement.

Figure 11 shows the comparison between the measurement and simulation results at $7.0 \mathrm{GHz}$ for $5 \times 5$ array. The measurement results for $4 \times 4$ array are not shown here. Due to the increase in gain, the patterns for the larger array show a better agreement with the simulations. The tolerance and error influences decrease as the number of the elements in the array increases. The measurements confirm the scanning capabilities of the suggested passive phasing technique.

The overall measured patterns are, however, different from the simulations. This can be explained by the following uncertainties. 


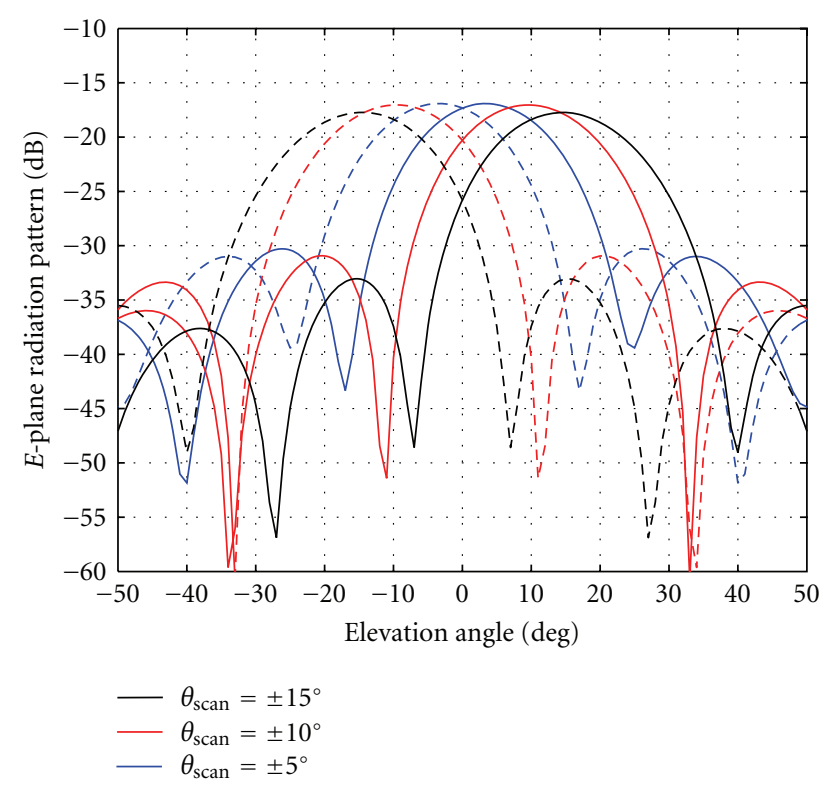

FIgURE 9: Simulated results for an $1 \times 6$ linear array demonstrating the beam scanning capabilities of an active MRA antenna.

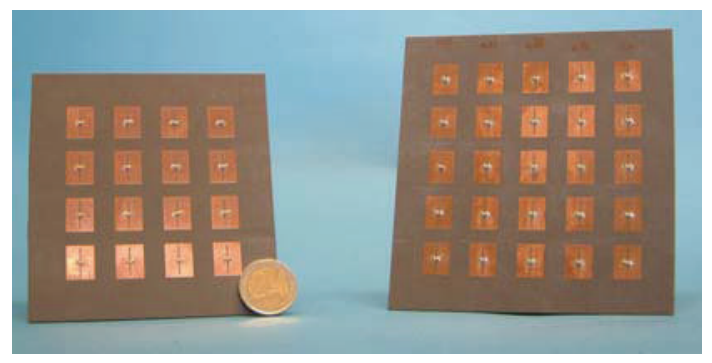

Figure 10: Manufactured $4 \times 4$ and $5 \times 5$ SMD capacitor-loaded MRA.

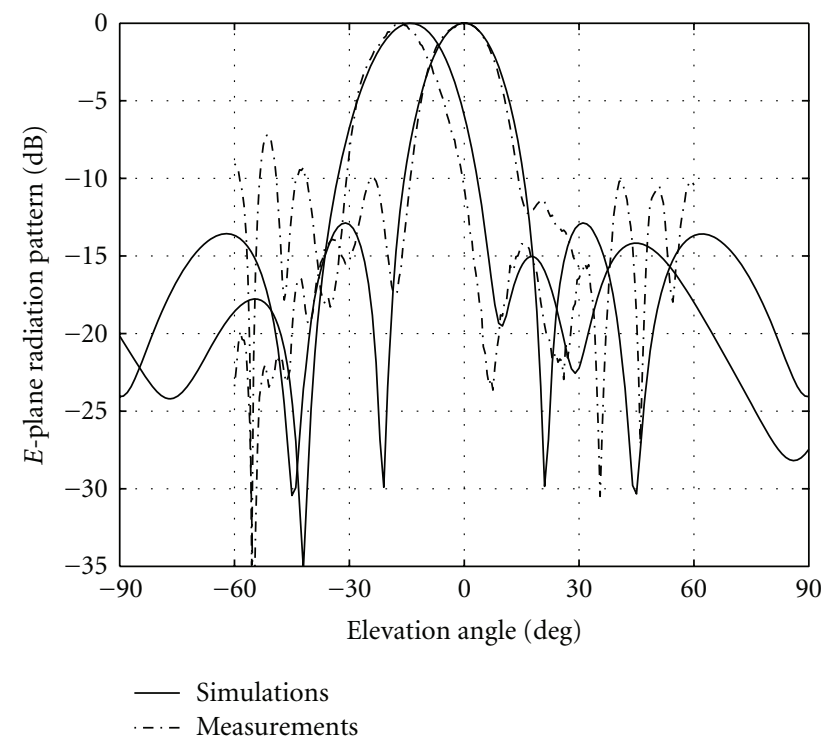

FIGURE 11: Comparison between the measurement- and simulation results for $5 \times 5$ SMD capacitor loaded MRA antenna. (i) Measurement set-up:

(a) feed: position, influence of the flens;

(b) alignment: the feed and receive antenna alignment was not ideal;

(c) plane wave: the array should be in the far field of the feed. For the measurement set-up, the inaccuracy of the plane wave illumination is not known.

(ii) Simulations: to model SMD a geometry consisting of a metal box capacitively loaded between the connection area and the center is used. The excitated surface currents are distributed on the metal box. In real terms the SMD is a ceramic multilayered capacitor with electrodes distributed in the medium. The model is a simplified leading to a difference in current distribution.

(iii) Tolerances and parasitic effects:

(a) tolerance: SMD has a tolerance of $\pm 0.25 \mathrm{pF}$. Extensive study is necessary to investigate the influences of such tolerance on the array performance;

(b) parasitic effects: in [19] the parasitic effects are treated in more detail. The SMD components are not ideal, introducing frequency dependent induction.

(iv) Manual placement:

(a) centre: the location of the SMD is inaccurate and the deviation is in the order of $\mathrm{mm}$. In the simulations the SMDs are exactly mounted on the centre of the slot and the distance between the connection points and the slot edge is the same for the both sides;

(b) soldering: the SMD are soldered onto the patch which introduce parasitic effects. The affect is not taken into account. The transition between the SMD and patch is assumed ideal for the simulations.

(v) Ground plane dimensions: the reflection of the ground plane influences the behavior of the antenna. Adjusting the dimension of the ground plane leads to change in the radiation pattern. Tuning using the ground plane dimensions is an empirical process leading to a satisfactory radiation pattern.

The measurements may be disappointing from an antenna point of view, they do, however, demonstrate the concept of beam scanning using capacitive loading.

6.2. Active. The technological process associated to the hollow MRA laminates is well established and manufacturing accuracy is very high. Moreover, for isolated elements it was necessary to metalize the via for bond wiring purposes. This is a well-developed technique in microstrip technology and has been adopted in this work. 


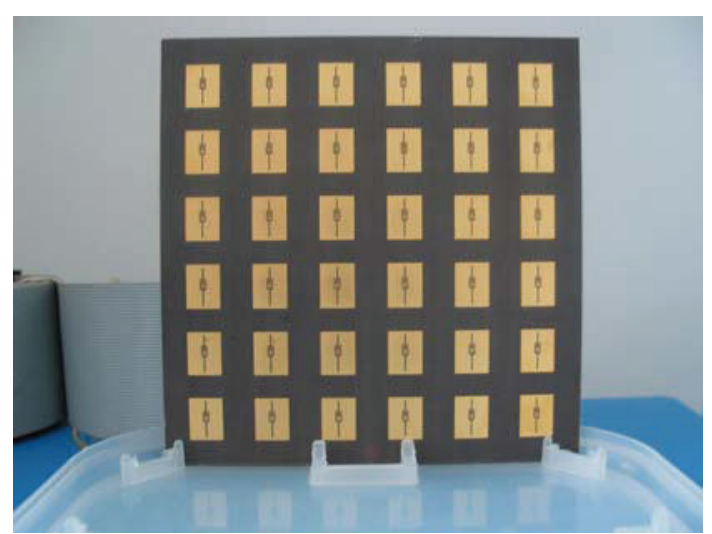

(a)

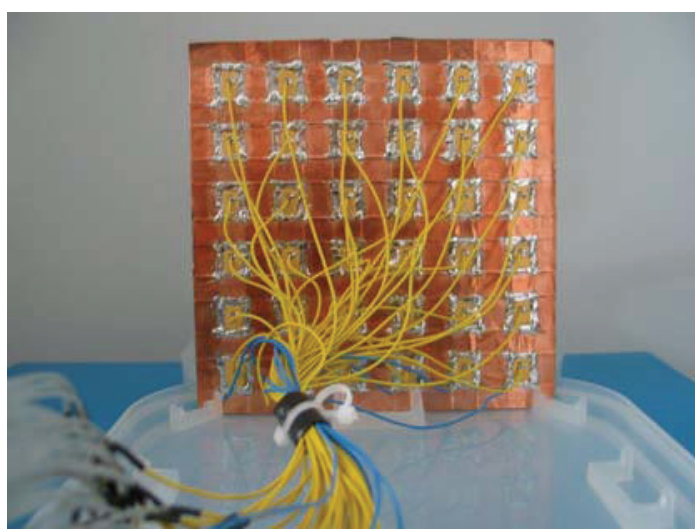

(b)

Figure 12: Manufactured active antennas. (a) front view; (b) rear view.

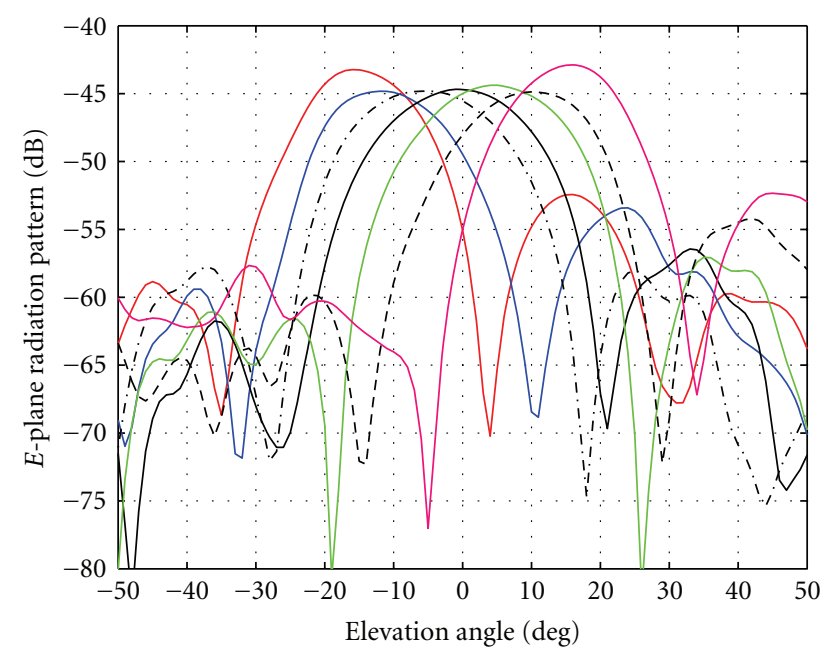

FIgURE 13: The measured radiation pattern at $6.15 \mathrm{GHz}$.

For bond wiring, vias with a diameter of $0.3 \mathrm{~mm}$ have been drilled in the PCB laminate and were made of golden material using bondable gold. The bond wires with diameter of $17 \mu \mathrm{m}$ are integrated using the "Wedge Wedge" process technique [20]. It has a Gaussian bell form with a height of $300 \mu \mathrm{m}$. The varactor chip with the thickness of $500 \mu \mathrm{m}$ is assembled on the top of the metalized island as it is presented in Figure 2, glued having a thickness of $60 \mu \mathrm{m}$. The glue is not a conductive material, and it is carefully injected and spread manually over the metallic island. The chip is subsequently mounted on the glue. The complete antenna is then kept in the oven for over two hours with a maximum temperature of $100^{\circ} \mathrm{C}$. The $6 \times 6$ planar integrated active hollowed MRA antenna that is depicted in Figure 12 was built in printed technology using high-frequency laminated material TLX-00620-C1/C1 from Taconic with a thickness of $1.57 \mathrm{~mm}$ and dielectric permittivity of $\epsilon_{r}=2.45$.

In order to block the leakage of the RF signal to the DC part, an SMD ceramic multilayer capacitor chip is integrated (soldered) on the bottom side of each elementary radiator. As depicted in Figure 12(b), the control signals include 36 wires for the biasing (yellow wires) and 6 lines for the grounding (blue wires) which have been soldered on the bottom side of each radiator in the array. Each column of the array requires one line for grounding.

6.3. Experimental Results. The experimental results presented in this paragraph were performed in Delft University Chamber for Antenna Test (DUCAT). The measurements in frequency domain are performed using the following equipment: a HP 81 PNWA (Personal Network Analyser) operating from $10 \mathrm{MHz}$ to $50 \mathrm{GHz}$, PCI-766 DAC (Digitalto-Analog converter) with an advanced multichannel analogue output board with optimised 16 channels each with full 16-bit resolution. The DAC was already available in the laboratory and in order to keep the costs low it was decided to use this one and not purchasing an extra DAC with more channels. The drawback is that in this case, a DAC channel is used to provide the information on the number of each individual antenna element simultaneously. The feed antenna was WR159 operational in $4.9 \mathrm{GHz} \leq f \leq$ $7.05 \mathrm{GHz}$ frequency band. A PC has been used to control and execute automatically the DAC output during the pattern measurements. The output of the DAC is via a ScSi and a 50 input 50 output universal adapter connected with 50 lines flat cable (length of $4 \mathrm{~m}$ ) to the array antenna inside the DUCAT. From the 50 lines of the flat cable, only 42 lines were used, 36 for the biasing and 6 for the ground signals. The radiation pattern of the manufactured antenna has been investigated in the $E$-plane at $f=6.15 \mathrm{GHz}$ and is depicted in Figure 13; scanning from $-15^{\circ} \leq \theta_{\text {scan }} \leq 15^{\circ}$ demonstrates the concept. The shift of $150 \mathrm{MHz}$ is due to the induction introduced by the bondwire which was not taken into the consideration in the simulations. Since it was not possible to tune each individual varactor chip on the array, it is not fully correct to compare the measured radiation patterns to the optimised numerical results. However, the measured and simulated radiation patterns for different scanning angles are depicted in Figure 14. The field patterns are shown in the E-plane only. The measurements are in good agreement with the simulations despite the significant difference between the theoretical model and the physical 


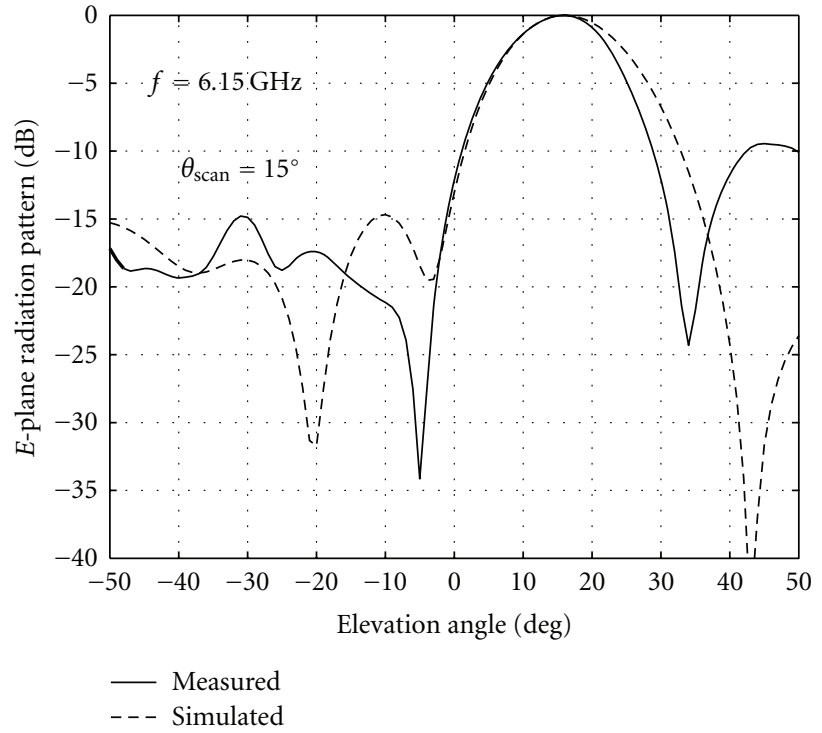

(a)

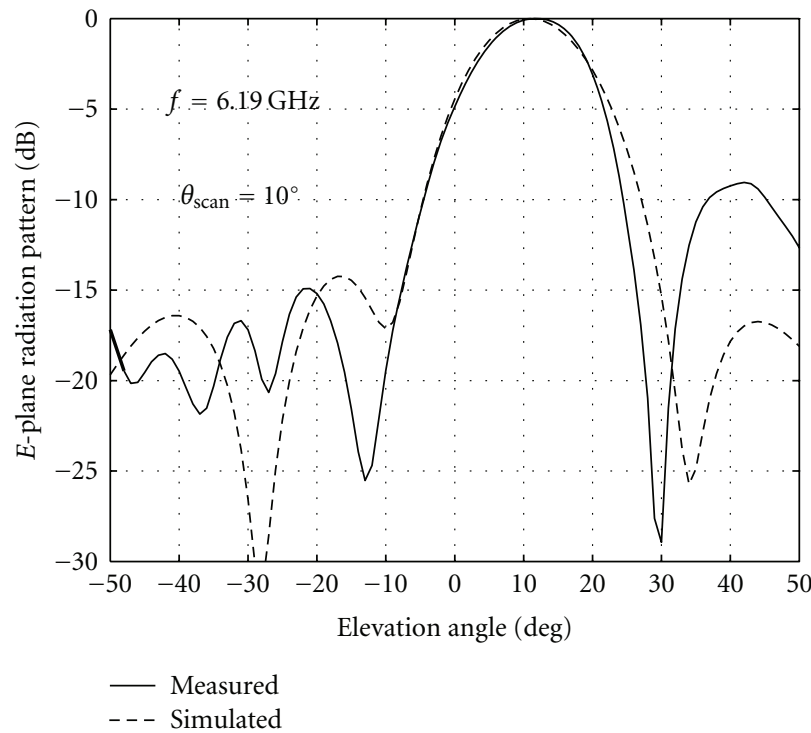

(b)

FIGURE 14: Measured and simulated radiation patterns of the active hollow array antenna at $f=6.15 \mathrm{GHz}$ for $\theta_{\text {scan }}=10^{\circ}, 15^{\circ}$.

realization. The differences are more visible at the larger scan angles.

\section{Conclusion}

This paper presents a passive and active phase shifting technique based on capacitive loading for reflectarray antennas. The reflectarray is designed at $7.0 \mathrm{GHz}$ using SMD capacitors. The commercially available SMD capacitors do not provide extensive array design possibilities; however, it is an unique method in creating phase-shift elements for reconfigurable antennas. An array of $5 \times 5$ is built, the elements of the array are integrated with SMD capacitors, and its radiation pattern is measured. The concept of scanning is demonstrated and there is a reasonable agreement between the theoretical and measurement results. A general design approach has been presented for active MRAs. The proposed active MRA consists of integrating hollow patches with tunable capacitors in the form of varactor diodes. Strategies for the design and manufacturing of active MRA's equipped with varactor chips as active element have been addressed. An effective computational solution incorporating a full-wave analysis of the active array has been presented. A complex measurement setup has been realized in DUCAT for performing radiation pattern measurements and steering each individual varactor chip. Measurements have been performed on an experimental active MRA. The concept of reconfigurable antenna using reflectarray is demonstrated. Considering the drawback of the DAC employed in the measurement setup, the experimental results and our numerical solution obtained by the full wave analysis are in good agreement. The design strategy that incorporates the numerical analysis of elementary radiators and active chips lends itself to several extensions. Firstly, it is observed that due to the realized capacitance versus voltage characteristics of the varactor, the scanning capability of $6 \times 6$ array is limited to elevation scan angles up to $\pm 18^{\circ}$. The operational frequency of the varactor opens the possibilities to examine array antennas at higher frequencies.

\section{References}

[1] R. D. Javor, X. D. Wu, and K. Chang, "Design and performance of a microstrip reflectarray antenna," IEEE Transactions on Antennas and Propagation, vol. 43, no. 9, pp. 932-939, 1995.

[2] M. Hajian, A. Coccia, and L. P. Ligthart, "Design, analysis and measurements of reflected phased array antennas at Ka-band using passive stubs," in Proceedings of the European Conference on Antennas and Propagation (EuCAP '06), Nice, France, 2006.

[3] J. Huang and R. J. Pogorzelski, "A ka-band microstrip reflectarray with elements having variable rotation angles," IEEE Transactions on Antennas and Propagation, vol. 46, no. 5, pp. 650-656, 1998.

[4] D. M. Pozar, S. D. Targonski, and H. D. Syrigos, "Design of millimeter wave microstrip reflectarrays," IEEE Transactions on Antennas and Propagation, vol. 45, no. 2, pp. 287-296, 1997.

[5] M. Hajian and N. F. Kiyani, "Design, analysis and measurements of reflectarray using variable length microstrip patch antennas at Ka-band," in Proceedings of the 18th Annual IEEE International Symposium on Personal, Indoor and Mobile Radio Communications (PIMRC '07), Athens, Greece, 2007.

[6] C. Trampuz, M. Hajian, and L. P. Ligthart, "Design, analysis and measurements of reflected phased array microstrip antennas at Ka-band, using hollow phasing," in Proceedings of the 36th European Microwave Conference Proceedings (EURAD '06), pp. 57-60, Manchester, UK, 2006.

[7] K. Buisman, L. C. N. De Vreede, L. E. Larson et al., "Lowdistortion, low-loss varactor-based adaptive matching networks, implemented in a silicon-on-glass technology," in Proceedings of the IEEE Radio Frequency Integrated Circuits Symposium (RFIC '05), pp. 389-392, June 2005.

[8] K. Buisman, L. C. N. De Vreede, L. E. Larson et al., “"Distortion-free" varactor diode topologies for RF adaptivity," 
in Proceedings of the IEEE MTT-S International Microwave Symposium, pp. 157-160, June 2005.

[9] D. Sievenpiper, J. Schaffner, R. Loo, G. Tangonan, S. Ontiveros, and R. Harold, "A tunable impedance surface performing as a reconfigurable beam steering reflector," IEEE Transactions on Antennas and Propagation, vol. 50, no. 3, pp. 384-390, 2002.

[10] J. P. Gianvittorio and Y. Rahmat-Samii, "Reconfigurable patch antennas for steerable reflectarray applications," IEEE Transactions on Antennas and Propagation, vol. 54, no. 5, pp. 13881392, 2006.

[11] C. won Jung, M. J. Lee, G. P. Li, and F. De Flaviis, "Reconfigurable scan-beam single-arm spiral antenna integrated with RF-MEMS switches," IEEE Transactions on Antennas and Propagation, vol. 54, no. 2, pp. 455-463, 2006.

[12] M. Riel and J. J. Laurin, "Design of an electronically beam scanning reflectarray using aperture-coupled elements," IEEE Transactions on Antennas and Propagation, vol. 55, no. 5, pp. 1260-1266, 2007.

[13] M. Hajian, Passive and Active Reconfigurable Microstrip Reflectarray Antennas, Delft University of Technology, 2007.

[14] Philips Components, "Surface mounted ceramic multilayer capacitors," Product Specification, Class 1, NP0 50V microwave series.

[15] P. Blondy, C. Champeaux, P. Tristant et al., "Applications of RF MEMS to tunable filters and matching networks," in Proceedings of the International Semiconductor Conference, pp. 111-116, October 2002.

[16] J. T. M. van Beek, M. H. W. M. van Delden, A. van Dijken et al., "High-Q integrated RF passives and micro-mechanical capacitors on silicon," in Proceedings of the BIPOLAR/BICMOS Circuits and Technology Meeting, pp. 147-150, September 2003.

[17] J. van Beek and M. van Delden, "Realization of high tunability barium strontium titanate thin films by RF magnetron sputtering," Applied Physics Letters, vol. 75, no. 20, pp. 3186-3188, 1999.

[18] K. Buisman, L. C. N. de Vreede, L. E. Larson et al., "A monolithic low-distortion low-loss silicon-on-glass varactor-tuned filter with optimised biasing," Microwave and Wireless Components Letters, vol. 17, no. 1, pp. 58-60, 2007.

[19] B. Kuijpers and M. Hajian, "Development of active microstrip reflectarray antennas using capacitive loading on a slotted patch," Internal report IRCTR-A-006-06, Delft, The Netherlands, 2006.

[20] W. C. Till and J. T. Luxon, Integrated Circuits: Materials, Devices and Fabrication, Prentice Hall, 1982. 

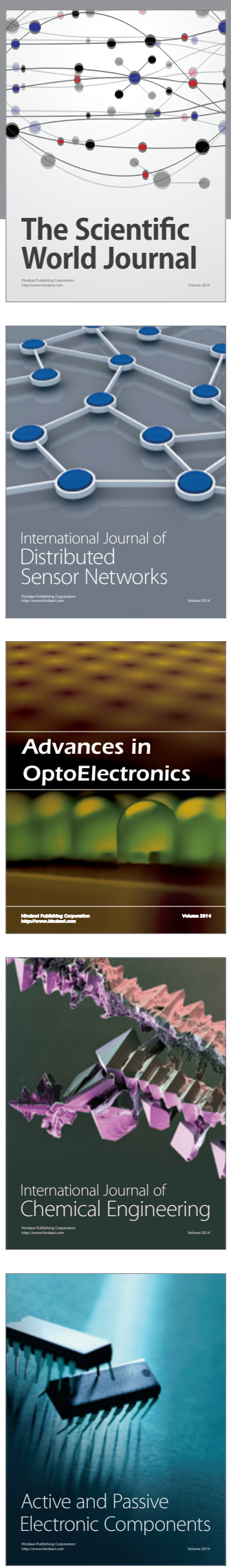
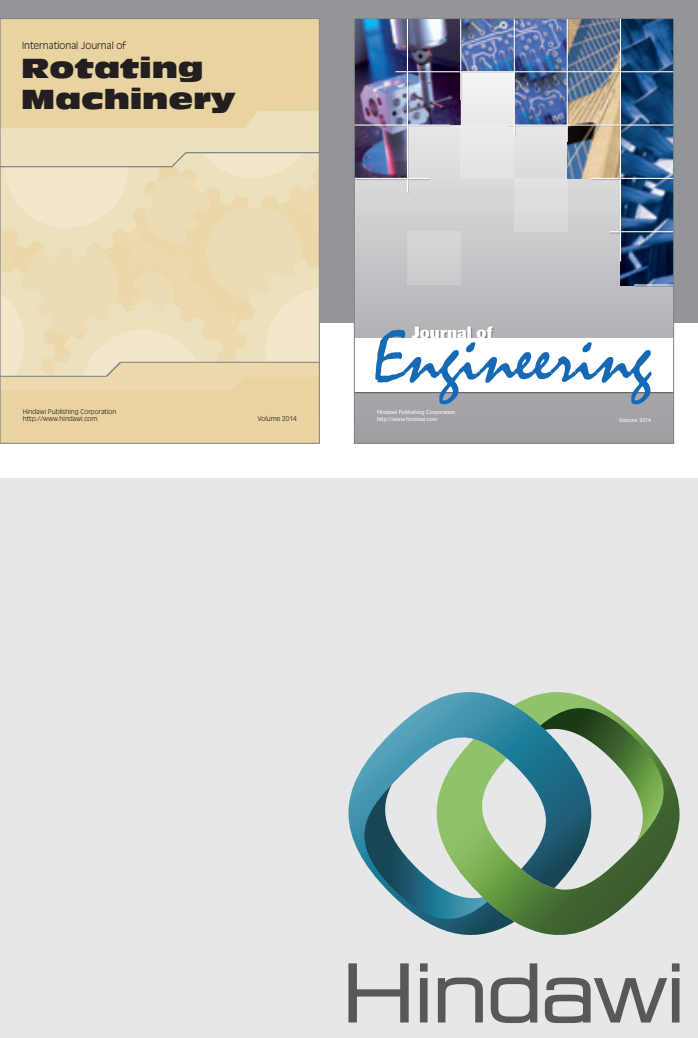

Submit your manuscripts at

http://www.hindawi.com
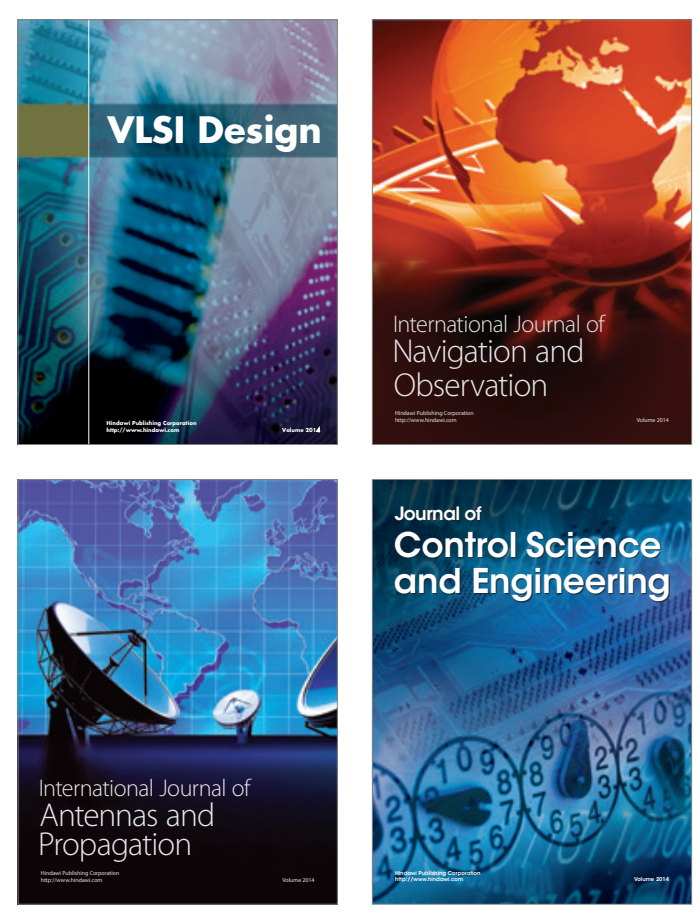
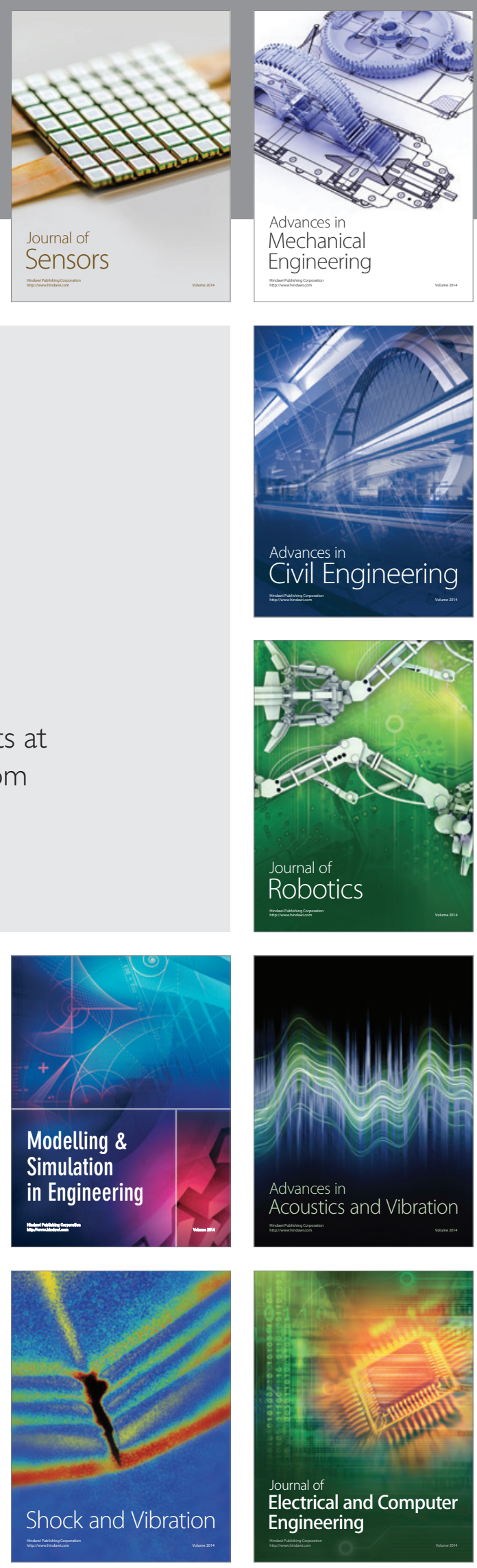